\title{
Comorbidity and Inflammatory Markers May Contribute to Predict Mortality of High-Risk Patients With Chronic Obstructive Pulmonary Disease Exacerbation
}

\author{
Yu Jin Kima, b, Byeongwoo Limª, b, Sun Young Kyung ${ }^{\mathrm{a}}$, Jeong-woong Park ${ }^{\mathrm{a}}$, \\ Sung Hwan Jeonga, c
}

\begin{abstract}
Background: Acute exacerbation of chronic obstructive pulmonary disease (COPD) causes not only an accelerated disease progression, but also an increased mortality rate. The purpose of this study was to analyze the factors associated with clinical features, comorbidities and mortality in patients at high risk for acute COPD exacerbation who had been hospitalized at least once in a year.
\end{abstract}

Methods: The study enrolled 606 patients who had been diagnosed with and were being treated for COPD at university affiliated hospital. Among them, there were 61 patients at high risk for acute exacerbation of COPD who had been hospitalized at least once in a year. A retrospective analysis was conducted to examine the factors affecting mortality. The analysis divided the patients into non-survivor and survivor groups, and reviewed their medical records for clinical aspects, comorbidities, pulmonary function tests and blood tests.

Results: In the high-risk group, the number of comorbidities at diagnosis $(\mathrm{P}=0.020)$ and the Charlson comorbidity index value $(\mathrm{P}=$ 0.018 ) were higher in the non-survivor group than in the survivor group. During hospitalization, the non-survivor group had a significantly higher neutrophil (\%) and a significantly lower lymphocyte (\%) in complete blood count. Under stable conditions, the high-sensitivity C-reactive protein (hsCRP) concentration in blood plasma and neutrophil (\%) were significantly higher $(\mathrm{P}=0.025$ and $\mathrm{P}=0.036)$, while the lymphocyte $(\%)$ was significantly lower $(\mathrm{P}=0.005)$ in the non-survivor group. A pulmonary function test revealed no statistically significant differences between the two groups.

Conclusion: The number of comorbidities, neutrophil (\%), lymphocyte (\%) in complete blood cell (CBC) and hsCRP in blood plasma concentration among the groups at high risk for COPD exacerbation

Manuscript accepted for publication May 13, 2016

aDivision of Pulmonology and Allergy, Department of Internal Medicine, Gachon University Gil Medical Center, Incheon, Korea

${ }^{\mathrm{b}}$ These authors contributed equally to this work

${ }^{\mathrm{c}}$ Corresponding Author: Sung Hwan Jeong, Division of Pulmonology, Department of Internal Medicine, Gachon University Gil Medical Center, Guwol1dong, Namdong-gu, Incheon, Korea. Email: jsw@gilhospital.com

doi: http://dx.doi.org/10.14740/jocmr2594w are associated with increased mortality.

Keywords: COPD; High risk of exacerbation; Comorbidity; Hospitalization

\section{Introduction}

Acute exacerbation of chronic obstructive pulmonary disease (COPD) refers to a severe deterioration in the condition of a COPD patient that requires a medication change due to their baseline respiratory symptoms exceeding the daily fluctuation range $[1,2]$. Patients with COPD frequently experience this acute exacerbation, which can reduce their quality of life and pulmonary function, while causing greater socioeconomic burden and accelerating disease progression [3, 4]. Previous studies reported that the number of acute COPD exacerbations had an influence on mortality. Other authors reported that the mortality rate ranged greatly from lower than $22 \%$ to $49 \%$, depending on the severity of the acute COPD exacerbation that caused the patient to be hospitalized [5, 6]. As shown, acute exacerbation of COPD has a major influence on mortality; therefore, the combined COPD assessment from Global Initiative from Chronic Obstructive Lung Disease (GOLD), published in 2011, recommended that patients who have been admitted at least once a year for acute exacerbation need to be classified as high risk (group C or D) and treated more aggressively [7]. It is very important to identify the risk factors associated with COPD exacerbation onset and recurrence. However, to our knowledge, studies of these high-risk groups experiencing at least once a year hospitalization are lacking in South Korea. Therefore, the authors of this study investigated the clinical features, comorbidities and mortality of high-risk patients who were diagnosed with COPD at Gachon University Gil Hospital and hospitalized at least once a year.

\section{Materials and Methods}

\section{Study design}

A retrospective survey was conducted on the medical records 
Table 1. Clinical Characteristics of All COPD Patients

\begin{tabular}{llll}
\hline Baseline characteristics & Exacerbation $(\mathbf{N})(\mathbf{N}=\mathbf{2 8 5})$ & Exacerbation $(\mathbf{Y})(\mathbf{N}=\mathbf{3 2 1})$ & P-value \\
\hline Male/female $(\mathrm{n})$ & $247 / 38$ & $266 / 55$ & 0.246 \\
Age $($ years $)$ & $70.07 \pm 8.98$ & $73.14 \pm 9.35$ & 0.000 \\
Height $(\mathrm{cm})$ & $164 \pm 7.34$ & $163 \pm 8.63$ & 0.121 \\
Weight $(\mathrm{kg})$ & $58.9 \pm 10.1$ & $56.5 \pm 10.4$ & 0.003 \\
Body mass index $\left(\mathrm{kg} / \mathrm{m}^{2}\right)$ & & & 0.019 \\
$\quad$ BMI $<18.5$ & 42 & 69 & 214 \\
$\quad 18.5 \leq$ BMI $<25$ & 192 & 38 & 0.002 \\
$\quad 25 \leq \mathrm{BMI}$ & 52 & $5,969.47 \pm 3,711.41$ & 0.007 \\
WBC $\left(10^{3} / \mathrm{mm}^{3}\right)$ & $8,035.63 \pm 3,145.68$ & $4.02 \pm 0.54$ & 0.984 \\
Serum albumin & $4.13 \pm 0.43$ & $1.31 \pm 1.52$ & 0.162 \\
CRP & $1.32 \pm 2.53$ & $33.58 \pm 11.96$ & 0.211 \\
Pulmonary hypertension & $31.04 \pm 20.83$ & $44.43 \pm 29.78$ & \\
Pack-years & $41.19 \pm 19.09$ & & \\
\hline
\end{tabular}

Values are presented as the mean \pm standard deviation unless otherwise indicated. P-values were generated by Student's $t$-tests for continuous variables and Chi-squared tests for categorical variables.

of patients who were diagnosed with COPD at Gachon University Gil Hospital between October 1999 and June 2013. First, patients with COPD were selected from the hospital's electronic medical record system according to the International Classification of Diseases (ICD) coding (ICD-9: 490-492, 494-496). Among these patients, those with the following risk factors were included in the study: smoking history and reduced pulmonary function (forced expiratory volume in $1 \mathrm{~s}$ $\left(\mathrm{FEV}_{1}\right)$ to forced vital capacity $(\mathrm{FVC})$ ratio $\left(\mathrm{FEV}_{1} / \mathrm{FVC}\right)$ lower than 0.7) [7]. Other lung diseases, such as asthma, lung cancer, bronchiectasis, active tuberculosis, and pleurisy, were excluded. Acute exacerbation of COPD was based on the GOLD guidelines and included outpatient visits and hospitalizations from acute events manifesting with exacerbation of respiratory symptoms that required a medication switch due to unusual changes $[7,8]$. The group that was at high risk for acute COPD exacerbation consisted of patients who had been hospitalized at least once for acute COPD exacerbation, per the GOLD guidelines [7]. The enrolled patients' age, gender, body weight, body mass index (BMI), smoking status, comorbidities, medications administered, follow-up period, and survival status were analyzed. The patients' comorbidities were defined as having already been diagnosed and being treated, while laboratory test result analyses used test results under stable conditions and from outpatient visit- or hospitalization-exacerbated conditions.

\section{Statistical analysis}

Data were expressed mean \pm standard deviation. Continuous data were done by the Student's $t$-test and categorical data were compared by the Pearson's Chi-square test. Differences were considered significant when the $\mathrm{P}$ value was less than 0.05 . Statistical analyzed were performed by IBM SPSS Sta- tistics 21 .

\section{Results}

Clinical characteristics, blood tests and pulmonary function test

A total of 606 patients were enrolled in the study, of which 321 were assigned to the exacerbation group. The mean age of the exacerbation group was 73.14 years, which was significantly higher than the mean of 70.7 years in the non-exacerbation group $(\mathrm{P}=0.000)$. Meanwhile, the mean body weight $(\mathrm{P}=$ $0.003)$ and BMI $(\mathrm{P}=0.019)$ were significantly lower in the exacerbation group. The laboratory test results indicated significantly lower white blood cell $(\mathrm{WBC})$ counts $(\mathrm{P}=0.002)$ and serum albumin concentrations $(\mathrm{P}=0.007)$ in the exacerbation group (Table 1).

Pulmonary function tests indicated the exacerbation group showed significantly lower $\mathrm{FVC}, \mathrm{FEV}_{1}, \mathrm{FEV}_{1} / \mathrm{FVC}$, and forced expiratory flow 25-75\% (FEF25-75) values while showing a significantly high at GOLD III and IV stage (Table 2 ). The most common comorbidity for all patients was cardiovascular disease, including hypertension and ischemic heart disease, while a significantly higher number of diabetes $(\mathrm{P}=$ $0.012)$ and benign prostatic hyperplasia $(\mathrm{P}=0.001)$ cases were found in the exacerbation group (Table 3 ).

Comparison of clinical characteristics and pulmonary function test between high-risk patients for COPD exacerbation

A total of 61 patients who had been hospitalized at least once 
Table 2. Pulmonary Function Test of All COPD Patients

\begin{tabular}{|c|c|c|c|}
\hline Variable & Exacerbation $(\mathrm{N})(\mathrm{N}=\mathbf{2 8 5})$ & Exacerbation $(\mathrm{Y})(\mathrm{N}=321)$ & P-value \\
\hline \multicolumn{4}{|c|}{ Pulmonary function test (post-bronchodilator) } \\
\hline $\mathrm{FVC}(\mathrm{L})$ & $2.82 \pm 1.07$ & $2.30 \pm 0.81$ & 0.000 \\
\hline $\mathrm{FVC}(\%)$ & $77.66 \pm 18.94$ & $67.43 \pm 2.68$ & 0.000 \\
\hline $\mathrm{FEV}_{1}(\mathrm{~L})$ & $1.45 \pm 0.60$ & $1.10 \pm 0.48$ & 0.000 \\
\hline FEV $(\%)$ & $58.34 \pm 20.93$ & $48.10 \pm 21.37$ & 0.000 \\
\hline $\mathrm{FEV}_{1} / \mathrm{FVC}(\%)$ & $51.48 \pm 11.38$ & $47.74 \pm 10.84$ & 0.000 \\
\hline FEF25-75 (\%) & $25.84 \pm 16.21$ & $21.83 \pm 14.89$ & 0.002 \\
\hline GOLD stage & & & 0.000 \\
\hline I & 43 & 33 & \\
\hline II & 144 & 99 & \\
\hline III & 83 & 125 & \\
\hline IV & 16 & 64 & \\
\hline
\end{tabular}

Values are presented as the mean \pm standard deviation unless otherwise indicated. P-values were generated by Student's $t$-tests for continuous variables and Chi-squared tests for categorical variables. FEV 1 : forced expiratory volume in $1 \mathrm{~s}$; FVC: forced vital capacity; FEF2575: forced expiratory flow at 25-75\%; GOLD stage: global initiative for chronic obstructive pulmonary disease stage.

in a year met the criteria for the high-risk group, equivalent to group $\mathrm{C}$ or $\mathrm{D}$ according to the combined COPD assessment criteria. Among these patients, 21 were in the non-survivor group and 40 were in the survivor group. There was no statistically significant difference in age, gender, or smoking history (pack-years) between the two groups. The mean observation period was 1,509.67 days, and there was no statistically significant difference between the non-survivor (1,448.38 days) and survivor $(1,541.85$ days $)$ groups $(\mathrm{P}=0.763)$. A comparison of the average number of annual acute exacerbation events between the two groups showed that the non-survivor group had a higher number of such events than the survivor group, but the difference was not statistically significant $(\mathrm{P}=$ 0.143). Moreover, there were no significant differences between the two groups with respect to the drug treatment in stable periods (inhaled short-acting anticholinergic, inhaled long-acting anticholinergic, inhaled long-acting $\beta 2$-agonist, inhaled long-acting $\beta 2$-agonist with inhaled corticosteroid, methylxanthine, systemic corticosteroid) used for treating
COPD (Table 4).

\section{Comparison of comorbidities and blood test between high- risk patients for COPD exacerbation}

Among the patients in the high-risk group whose comorbidity at the time of diagnosis was identifiable, the non-survivor group had a higher number of comorbidities than the survivor group ( 2.83 vs. $1.88, \mathrm{P}=0.020)$, with the most common comorbidity being cardiovascular disease, including hypertension and myocardial infarction. A severity assessment based on the Charlson comorbidity index (CCI) [9] showed that scores of 1 (moderate), 2 (severe) and 3 or greater (very severe) were seen in 16, 14, and two patients in the survivor group, respectively, and five, six, and seven patients in the non-survivor group, respectively $(\mathrm{P}=0.018)$ (Table 5$)$. In comparison to the moderate group (score of 1 ), the mortality rate ratio (MRR) was $1.543(95 \% \mathrm{CI}: 0.389-6.115, \mathrm{P}=0.537)$ in the severe

Table 3. Comorbidities of All COPD Patients

\begin{tabular}{llll}
\hline Variable & Exacerbation $(\mathbf{N})(\mathbf{N}=\mathbf{2 8 5})$ & Exacerbation $(\mathbf{Y})(\mathbf{N}=\mathbf{3 2 1})$ & P-value \\
\hline Comorbidities & & & 0.012 \\
$\quad$ Diabetes mellitus & 30 & 55 & 0.289 \\
Hypertension & 98 & 118 & 0.001 \\
Benign prostate hypertrophy & 14 & 40 & 0.299 \\
Ischemic heart disease & 37 & 36 & 0.432 \\
Cerebrovascular accident & 9 & 12 & 0.153 \\
Lung cancer & 4 & 1 & 0.242 \\
Other cancer & 16 & 13 & \\
\hline
\end{tabular}

Other cancer: stomach cancer, bladder cancer, breast cancer, prostate cancer, ovary cancer. 
Table 4. Follow-Up Time and Medications of High-Risk Group Who Survived or Died During Follow-Up

\begin{tabular}{|c|c|c|c|c|}
\hline & All patients $(\mathrm{N}=61)$ & Non-survivor $(\mathrm{N}=\mathbf{2 1})$ & Survivor $(N=40)$ & P-value \\
\hline Follow-up time (days) & $1,509.67$ & $1,448.38$ & $1,541.85$ & 0.763 \\
\hline Number of AE* (years) & 1.46 & 1.66 & 1.35 & 0.143 \\
\hline Short acting anticholinergic** & 12 & 5 & 7 & 0.618 \\
\hline Long acting anticholinergic** & 36 & 11 & 25 & 0.340 \\
\hline Theophylline & 40 & 14 & 26 & 0.914 \\
\hline Doxophylline & 23 & 6 & 17 & 0.235 \\
\hline Systemic corticosteroid & 32 & 14 & 18 & 0.160 \\
\hline
\end{tabular}

AE: acute exacerbation. *Inhaler.

group (score of 2) and 12.600 (95\% CI: $1.966-80.757, \mathrm{P}=$ 0.008 ) in the very severe group (score of 3 or greater). Diabetes was found in six patients in the non-survivor group and 15 patients in the survivor group. Five patients in the survivor group had benign prostatic hypertroplasia, whereas no patients in the non-survivor group had it. Cancer was found in five patients in the non-survivor group (stomach cancer with bladder cancer, lung cancer, breast cancer, prostate cancer, ovarian cancer), which was significantly higher than the one patient with cancer (prostate cancer) found in the survivor group $(\mathrm{P}=$ 0.015) (Table 5).

The two groups did not have differences in erythrocyte sedimentation rate (ESR), high-sensitivity $\mathrm{C}$-reactive protein (hsCRP) concentration, or total white blood cell (WBC) during hospitalization, but the non-survivor group had a significantly higher neutrophil percentage $(\mathrm{P}=0.025)$ and a significantly lower lymphocyte percentage $(\mathrm{P}=0.011)$ in complete blood cell (CBC). Especially in stable conditions, the non-survivor group had a significantly higher hsCRP $(\mathrm{P}=0.025)$ concentration and neutrophil percentage $(\mathrm{P}=0.036)$ in $\mathrm{CBC}$, and a significantly lower lymphocyte percentage $(0.005)$ in $\mathrm{CBC}$ than the survivor group (Table 6).

\section{Discussion}

Because existing studies had reported that acute exacerbation of COPD was the primary cause of increased mortality, GOLDclassified patients admitted at least once a year for acute exacerbation of COPD were added to the group at high risk for COPD exacerbation (group C or D) $[3,7,10]$. Therefore, it is necessary to understand these patients' characteristics and administer active care; however, to our knowledge, studies of patients at high risk for COPD exacerbation are still limiting. This study is significant because it studied the factors that induce mortality in patients at high risk for COPD exacerbation.

Previous study reported that comorbidity was associated with the survival of patients with COPD [11]. Many studies also reported that comorbidity, particularly cardiovascular disease, was associated with the survival of patients with COPD $[10,12-14]$. In our study, exacerbation was closely related with higher age, lower BMI, lower albumin and lower pulmonary

Table 5. Comorbidities of High-Risk Group Who Survived or Died During Follow-Up

\begin{tabular}{|c|c|c|c|c|}
\hline & All patients $(\mathrm{N}=52)$ & Non-survivor $(\mathrm{N}=18)$ & Survivor $(\mathbf{N}=34)$ & P-value \\
\hline Number of comorbidities & 2.21 & 2.83 & 1.88 & 0.020 \\
\hline \multicolumn{5}{|l|}{ Comorbidities } \\
\hline Hypertension & 23 & 9 & 14 & 0.542 \\
\hline Ischemic heart disease & 3 & 2 & 1 & 0.272 \\
\hline Benign prostate hypertrophy & 5 & 0 & 5 & 0.150 \\
\hline Diabetes mellitus & 21 & 6 & 15 & 0.451 \\
\hline Cancer & 6 & 5 & 1 & 0.015 \\
\hline Charlson comorbidity index & & & & 0.018 \\
\hline 1 (moderate) & 23 & 5 & 18 & \\
\hline 2 (severe) & 20 & 6 & 14 & \\
\hline 3 (very severe) & 9 & 7 & 2 & \\
\hline
\end{tabular}

Cancer: stomach cancer with bladder cancer, lung cancer, breast cancer, prostate cancer, ovary cancer. 
Table 6. ESR, CRP and CBC of High-Risk Group Who Survived or Died During Follow-Up

\begin{tabular}{|c|c|c|c|c|}
\hline & All patients & Non-survivor & Survivor & P-value \\
\hline \multicolumn{5}{|l|}{ Hospitalization } \\
\hline ESR & 34.1370 & 31.9371 & 35.8970 & 0.532 \\
\hline hsCRP & 6.6225 & 6.4681 & 6.7386 & 0.844 \\
\hline $\operatorname{WBC}\left(10^{3} / \mathrm{mm}^{3}\right)$ & 11.5505 & 11.1554 & 11.8367 & 0.499 \\
\hline Neutrophil (\%) & 73.7187 & 76.7963 & 71.4901 & 0.025 \\
\hline Neutrophil count & $8,650.7538$ & $8,705.4729$ & $8,611.1296$ & 0.917 \\
\hline Lymphocyte (\%) & 16.0222 & 13.5863 & 17.7863 & 0.011 \\
\hline Lymphocyte count & $1,772.5236$ & $1,450.1176$ & $2,005.9904$ & 0.003 \\
\hline \multicolumn{5}{|l|}{ Stable } \\
\hline ESR & 24.3009 & 22.9476 & 25.0903 & 0.798 \\
\hline hsCRP & 1.0400 & 1.8273 & 0.5469 & 0.025 \\
\hline WBC $\left(10^{3} / \mathrm{mm}^{3}\right)$ & 9.3930 & 10.2663 & 8.9251 & 0.174 \\
\hline Neutrophil (\%) & 65.4005 & 70.0139 & 62.9289 & 0.036 \\
\hline Neutrophil count & $6,310.6560$ & $7,197.2366$ & $5,835.7021$ & 0.107 \\
\hline Lymphocyte (\%) & 23.4416 & 18.4410 & 25.9657 & 0.005 \\
\hline Lymphocyte count & $2,056.1535$ & $1,890.0601$ & $2,145.1321$ & 0.201 \\
\hline
\end{tabular}

ESR: erythrocyte sedimentation rate; hsCRP: high-sensitivity C-reactive protein.

function. The overall number of comorbidities was significantly associated with mortality $(\mathrm{P}=0.020)$. A comparison of the two groups (non-survivors vs. survivors), classified by severity according to the CCI showed a significant difference between the groups $(\mathrm{P}=0.018)$. The MRR of the very severe group relative to the moderate group was 12.600 (95\% CI: 1.966 $80.757, \mathrm{P}=0.008)$, which was consistent with findings from previous studies that an increase in the CCI was associated with a less favorable prognosis [15]. Plasma CRP concentration and neutrophil count in $\mathrm{CBC}$, which have been studied extensively, are inflammatory biomarkers of COPD that can be tested economically and easily through a blood test in a clinical setting [16]. Previous reports have indicated that WBC count, along with CRP, fibrinogen, and TNF- $\alpha$ concentrations are generally elevated in patients with COPD [3, 16, 17]. It was reported that when COPD is stable, CRP is proportional to age, weight, dyspnea, and quality of life, while being inversely proportional to motor ability and FEV1 $[18,19]$. In a study by Hana and colleagues, CRP concentration and neutrophil count were significantly elevated in hospitalized exacerbation cases [3]. Other author also reported that CRP concentration and neutrophil count were significantly higher in severe COPD exacerbation cases than in stable COPD cases [16]. In a study by Man and colleagues that examined the relationship between CRP and the mortality rate attributed to COPD, serum CRP concentration was a significant predictive factor of the mortality rate; it also increased the mortality rate attributed to cancer and cardiovascular diseases [20]. In a study by de Torres and colleagues that examined 218 patients with moderate to very severe COPD under stable conditions, serum CRP concentrations were higher in the mortality group but the difference was not statistically significant [21]. In the present study, which examined patients at high risk for acute exacerbation of COPD,
CRP concentrations measured under stable conditions were significantly higher in the non-survivor group $(\mathrm{P}=0.025)$. This study has some limitations. This study was conducted in one university hospital. Inflammatory biomarkers such as hsCRP concentration and WBC count were being measured, but other inflammation-related biomarkers, such as interleukin (IL)-6, IL-8, and TNF- $\alpha$ were not sufficiently investigated. Through the findings in this study, the researchers were able to identify the total number of comorbidities at the time of diagnosis and the hsCRP concentration measured under stable conditions as potential predictive factors for mortality in patients who were hospitalized for acute exacerbation of COPD.

In conclusion, patients at high risk for acute COPD exacerbation need to be diagnosed for comorbidities and administered thorough treatment and management of such comorbidities. It is believed that regular measurements of systemic inflammatory indicators, including hsCRP, neutrophils, and lymphocytes, during follow-up observations will help predict a patient's prognosis. A large-scale prospective study on this subject matter is necessary.

\section{References}

1. Celli BR, Barnes PJ. Exacerbations of chronic obstructive pulmonary disease. Eur Respir J. 2007;29(6):1224-1238.

2. Rodriguez-Roisin R. Toward a consensus definition for COPD exacerbations. Chest. 2000;117(5 Suppl 2):398S401S.

3. Mullerova H, Maselli DJ, Locantore N, Vestbo J, Hurst JR, Wedzicha JA, Bakke P, et al. Hospitalized exacerbations of COPD: risk factors and outcomes in the ECLIPSE cohort. Chest. 2015;147(4):999-1007. 
4. Suzuki M, Makita H, Ito YM, Nagai K, Konno S, Nishimura M. Clinical features and determinants of COPD exacerbation in the Hokkaido COPD cohort study. Eur Respir J. 2014;43(5):1289-1297.

5. Seemungal TA, Donaldson GC, Paul EA, Bestall JC, Jeffries DJ, Wedzicha JA. Effect of exacerbation on quality of life in patients with chronic obstructive pulmonary disease. Am J Respir Crit Care Med. 1998;157(5 Pt 1):14181422.

6. Soler-Cataluna JJ, Martinez-Garcia MA, Roman Sanchez P, Salcedo E, Navarro M, Ochando R. Severe acute exacerbations and mortality in patients with chronic obstructive pulmonary disease. Thorax. 2005;60(11):925-931.

7. Vestbo J, Hurd SS, Rodriguez-Roisin R. The 2011 revision of the global strategy for the diagnosis, management and prevention of COPD (GOLD) - why and what? Clin Respir J. 2012;6(4):208-214.

8. Lin J, Xu Y, Wu X, Chen M, Lin L, Gong L, Feng J. Risk factors associated with chronic obstructive pulmonary disease early readmission. Curr Med Res Opin. 2014;30(2):315-320.

9. Charlson ME, Pompei P, Ales KL, MacKenzie CR. A new method of classifying prognostic comorbidity in longitudinal studies: development and validation. J Chronic Dis. 1987;40(5):373-383.

10. Piquet J, Chavaillon JM, David P, Martin F, Blanchon F, Roche N. High-risk patients following hospitalisation for an acute exacerbation of COPD. Eur Respir J. 2013;42(4):946-955.

11. Antonelli Incalzi R, Fuso L, De Rosa M, Forastiere F, Rapiti E, Nardecchia B, Pistelli R. Co-morbidity contributes to predict mortality of patients with chronic obstructive pulmonary disease. Eur Respir J. 1997;10(12):27942800.

12. Fuso L, Incalzi RA, Pistelli R, Muzzolon R, Valente S, Pagliari G, Gliozzi F, et al. Predicting mortality of patients hospitalized for acutely exacerbated chronic obstructive pulmonary disease. Am J Med. 1995;98(3):272-277.

13. Jones PW, Nadeau G, Small M, Adamek L. Characteris- tics of a COPD population categorised using the GOLD framework by health status and exacerbations. Respir Med. 2014;108(1):129-135.

14. Bhatt SP, Dransfield MT. Chronic obstructive pulmonary disease and cardiovascular disease. Transl Res. 2013;162(4):237-251.

15. Jacobs DR, Jr., Kroenke C, Crow R, Deshpande M, Gu DF, Gatewood L, Blackburn H. PREDICT: A simple risk score for clinical severity and long-term prognosis after hospitalization for acute myocardial infarction or unstable angina: the Minnesota heart survey. Circulation. 1999;100(6):599-607.

16. Agusti A, Edwards LD, Rennard SI, MacNee W, TalSinger R, Miller BE, Vestbo J, et al. Persistent systemic inflammation is associated with poor clinical outcomes in COPD: a novel phenotype. PLoS One. 2012;7(5):e37483.

17. Stanojkovic I, Kotur-Stevuljevic J, Spasic S, Milenkovic B, Vujic T, Stefanovic A, Ivanisevic J. Relationship between bone resorption, oxidative stress and inflammation in severe COPD exacerbation. Clin Biochem. 2013;46(16-17):1678-1682.

18. de Torres JP, Cordoba-Lanus E, Lopez-Aguilar C, Muros de Fuentes M, Montejo de Garcini A, Aguirre-Jaime A, Celli BR, et al. C-reactive protein levels and clinically important predictive outcomes in stable COPD patients. Eur Respir J. 2006;27(5):902-907.

19. Pinto-Plata VM, Mullerova H, Toso JF, Feudjo-Tepie M, Soriano JB, Vessey RS, Celli BR. C-reactive protein in patients with COPD, control smokers and non-smokers. Thorax. 2006;61(1):23-28.

20. Man SF, Connett JE, Anthonisen NR, Wise RA, Tashkin DP, Sin DD. C-reactive protein and mortality in mild to moderate chronic obstructive pulmonary disease. Thorax. 2006;61(10):849-853.

21. de Torres JP, Pinto-Plata V, Casanova C, Mullerova $\mathrm{H}$, Cordoba-Lanus E, Muros de Fuentes M, AguirreJaime A, et al. C-reactive protein levels and survival in patients with moderate to very severe COPD. Chest. 2008;133(6):1336-1343. 